\title{
Espiral do conhecimento em frameworks de gestão do conhecimento: o caso de duas organizações em Portugal
}

\section{Mírian Oliveira}

Doutora em Administração. Professora do Programa de Pós-Graduação em Administração da Faculdade de Administração, Contabilidade e Economia da PUCRS (Brasil)

Grace Vieira Becker

Doutora em Administração. Professora do Programa de Pós-Graduação em Administração da Faculdade de Administração, Contabilidade e Economia da PUCRS (Brasil)

Cristiane Drebes Pedron

Doutora em Gestão. Professora do Instituto Superior de Economia e Gestão da Universidade Técnica de Lisboa (Portugal)

Felipe Dall'Igna

Mestre em Administração pelo Programa de PósGraduação em Administração da Faculdade de Administração, Contabilidade e Economia da PUCRS (Brasil)

O objetivo é analisar como os frameworks de implementação de Gestão do Conhecimento (GC) contemplam as formas de conversão do conhecimento. O método utilizado foi o estudo de caso em duas organizações que atuam em Portugal. Os frameworks focam nos aspectos relacionados com as condições para a implementação da GC e não nos modos de conversão do conhecimento. Concluiu-se que uma organização possui maior foco na externalização e outra organização possui um equilíbrio entre os modos de conversão.

Palavras-chave: Gestão do conhecimento; Espiral do conhecimento. 


\section{Knowledge Spiral in Knowledge Management Frameworks: the Case of Two Organizations in Portugal}

This research goal is to analyze how knowledge management implementation framework contemplates the knowledge spiral. The research method adopted was an exploratory multiple case study in two Portugal organizations. The main result is: frameworks focus on the aspects related with the implementation condition (support of high administration, culture, etc.), instead of knowledge spiral. One organization focus on the externalization, and the other organization has an equilibrium among externalization, combination, socialization, internalization.

Keywords: Knowledge management; Knowledge spiral.

Recebido em 24.06.2009 Aceito em 18.10.2010

\section{Introdução}

O interesse pela Gestão do Conhecimento (GC) é impulsionado nas organizações pela busca de vantagem competitiva, considerando um ambiente globalizado, com alta competitividade, constante mudança na demanda dos clientes, entre outros aspectos. Nesta pesquisa, GC é entendida como um processo que visa à criação, armazenamento, disseminação e utilização do conhecimento, integrando pessoas, processos e tecnologias, alinhados com os objetivos da organização, considerando fontes de conhecimento internas e externas à organização.

A GC em uma organização envolve as quatro formas de conversão do conhecimento (socialização, externalização, combinação e internalização). O conjunto dessas quatro formas é conhecido como espiral do conhecimento (TAKEUCHI; NONAKA, 2008). Para que a implementação da GC em uma organização tenha êxito, necessita contemplar essas formas de conversão do conhecimento (NONAKA, 1994).

Um framework que oriente a implementação da GC nas organizações, poderá permitir uma visão integrada sobre o conhecimento na organização e salientar as práticas associadas à respectiva gestão (HOLSAPPLE; JOSHI, 2002; WONG; ASPINWALL, 2004). Segundo Wong e Aspinwall (2004, p. 95), um framework para a implementação de GC é "uma estrutura ou um conjunto de princípios norteadores, os quais são descritos de tal forma que forneçam orientação e direção em como conduzir a Gestão do Conhecimento numa organização". Tendo em vista esses conteúdos, o objetivo desta pesquisa é analisar como os frameworks de implementação de GC contemplam a espiral do conhecimento. 


\section{Espiral do conhecimento e os frameworks de implementação da GC}

Esta seção descreve os quatros modos de conversão do conhecimento da espiral do conhecimento (2.1), os frameworks de implementação de GC (2.2 a 2.15) e a comparação dos frameworks e a relação com as quatro formas de conversão do conhecimento (2.16).

\subsection{Espiral do conhecimento}

O conhecimento tácito é composto por elementos técnicos (know-how e habilidades) e cognitivos (modelo mental, tal como paradigmas e crenças), sendo difícil de ser formalizado e transmitido (TAKEUCHI; NONAKA, 2008). Já o conhecimento explícito é aquele que pode ser facilmente codificado, podendo ser representado, armazenado, compartilhado e efetivamente aplicado (TAKEUCHI; NONAKA, 2008).

A socialização tem como objetivo criar e compartilhar conhecimento tácito através da interação entre as pessoas, ocorrendo a conversão do conhecimento tácito para o tácito. Nesse modo, a interação é de indivíduo para indivíduo (TAKEUCHI; NONAKA, 2008) e ocorre, geralmente, em reuniões informais (ISHIKURA, 2008). A socialização também pode ocorrer através da observação, imitação e da prática (NONAKA; TAKEUCHI, 2008).

A externalização tem 0 intuito de converter o conhecimento tácito em explícito. Nesse modo de conversão o conhecimento passa do indivíduo para o grupo (TAKEUCHI; NONAKA, 2008). "O conhecimento tácito torna-se explícito, tomando a forma de metáforas, analogias, conceitos, hipóteses ou modelos" (NONAKA; TAKEUCHI, 2008, p. 62).

A combinação tem como objetivo sistematizar o conhecimento explícito, ocorrendo a conversão de explícito para explícito. Nesse caso o conhecimento passa do grupo para a organização (TAKEUCHI; NONAKA, 2008). "Este modo de conversão de conhecimento envolve a combinação de diferentes corpos de conhecimento explícito" (NONAKA; TAKEUCHI, 2008, p. 65).

A internalização tem 0 intuito de converter o conhecimento explícito em tácito. Nesse modo, o conhecimento passa da organização para o indivíduo (TAKEUCHI; NONAKA, 2008). Esse modo está relacionado com o "aprender fazendo" (ISHIKURA, 2008).

\subsection{Wiig, De Hoog e Van Der Spek (1997)}

O framework proposto por Wiig, Hoog e Spek (1997) apresenta quatro atividades: revisão, conceitualização, reflexão e ação. A atividade de revisão compara o passado com o presente, ou seja, monitora e avalia o desempenho da organização sob a perspectiva da GC. Um programa de lições aprendidas é indicado para o monitoramento. A avaliação serve para indicar se as ações estão conduzindo a organização para os resultados almejados. A atividade de conceitualização compreende o mapeamento do conhecimento na organização e a análise dos seus pontos fortes e fracos. Os resultados dessa atividade são fundamentais para a tomada de decisão na atividade seguinte, ou seja, a 
reflexão. A atividade de reflexão desenvolve um plano de melhorias necessárias, considerando os riscos da implementação do mesmo. Essa atividade abrange a seleção e o detalhamento das melhorias, considerando tempo, orçamento, produto gerado, pessoas envolvidas, controle da qualidade, responsabilidades e riscos. A atividade de ação coloca o plano em prática, abrangendo a consolidação, combinação, distribuição e desenvolvimento do conhecimento.

O framework não menciona especificamente a espiral do conhecimento, mas, através das suas atividades é possível identificar indiretamente os quatro modos de conversão do conhecimento: socialização - na revisão através da discussão das pessoas sobre as lições aprendidas; externalização - através da documentação das lições aprendidas e do mapeamento do conhecimento; combinação - quando as lições aprendidas por cada grupo são combinadas, gerando um novo conhecimento e é realizado o mapeamento do conhecimento; internalização - através de cada indivíduo, ao acessar as lições aprendidas e na geração de novos conhecimentos tácitos. A ênfase ao conhecimento tácito ou explícito irá depender dos pontos fortes e fracos identificados através do mapeamento do conhecimento na organização.

\subsection{Liebowitz e Beckman (1998)}

O framework criado por Liebowitz e Beckman (1998 apud LIEBOWITZ, 1999) considera 6 elementos e um processo com 8 estágios. Os elementos apresentados pelos autores são: suporte da alta administração, tanto financeiro quanto moral; definição de uma estratégia, considerando o ambiente interno e externo; definição de um responsável pela GC na organização e uma equipe; escolha das ferramentas a serem utilizadas; definição de um sistema para motivar a participação dos funcionários; e a construção de uma cultura de partilha na organização.

Os estágios para o processo de GC descritos por Liebowitz e Beckman (1998 apud LIEBOWITZ, 1999, p. 37) são:

identificação - determinar as competências, e domínios do conhecimento; captura - formalizar o conhecimento existente; seleção - resolver conhecimento conflitante; armazenamento - colocar a memória organizacional em um repositório; compartilhamento - distribuir o conhecimento automaticamente para os usuários baseado no interesse e no trabalho de cada um; aplicação - recuperar e usar o conhecimento; criação - descobrir novos conhecimentos; venda desenvolver novos produtos e serviços baseados no conhecimento.

Os elementos apresentados pelos autores impactam nos modos de conversão do conhecimento. Por exemplo, a ferramenta escolhida para a GC pode privilegiar a interação entre os indivíduos, permitindo a socialização mesmo entre pessoas distantes geograficamente; pode também facilitar a documentação do conhecimento (externalização e combinação), assim como facilitar a recuperação do conhecimento auxiliando na internalização. Já os estágios propostos contemplam, indiretamente, três modos de conversão do conhecimento, que são externalização, combinação e internalização. Por exemplo, a externalização pode ser associada à captura, ou seja, formalização do conhecimento existente, e no armazenamento; a combinação pode ser percebida na seleção, onde são 
resolvidos conhecimentos conflitantes, e no armazenamento; a internalização pode ocorrer na venda, onde novos produtos e serviços são desenvolvidos com base no conhecimento.

\subsection{Gore e Gore (1999)}

O framework apresentado por Gore e Gore (1999) considera como ponto inicial a formulação de uma visão pela alta administração. Segundo os autores, provavelmente esse é o aspecto mais crítico para a GC. O segundo aspecto a ser considerado é a análise do uso do conhecimento explícito existente, considerando o seu fluxo na organização. O terceiro aspecto consiste na captura de novos conhecimentos explícitos, a partir da análise de processos organizacionais. 0 quarto aspecto é a externalização do conhecimento tácito, estimulado pelo trabalho em equipe, comunidades de prática, entre outros. O quinto aspecto é assegurar que o novo conhecimento organizacional seja utilizado na organização.

Esse framework aborda diretamente o conhecimento tácito e explícito, sendo possível verificar os modos de conversão presentes, indiretamente. Em relação à socialização, os autores mencionam o trabalho em equipe e as comunidades de prática. A externalização também pode ser visualizada através das comunidades de prática, onde o conhecimento tácito dos indivíduos é compartilhado e documentado para um determinado grupo. Os autores tratam a combinação ao considerar a análise do conhecimento explícito existente. Por fim, a internalização pode ser observada pela utilização do conhecimento organizacional nas atividades da organização, podendo gerar novos conhecimentos tácitos.

\subsection{McCampbell, Clare e Gitters (1999)}

O framework proposto por McCampbell, Clare e Gitters (1999, p. 179) é formado por 12 etapas, que são:

a) formar aliança - obter o suporte da alta administração; b) comunicar a visão - incorporar a mensagem nas atividades diárias da organização; c) estabelecer equipes - criar equipes para avaliar as necessidades; d) analisar as necessidades - conduzir avaliações das necessidades; e) identificar e adquirir conhecimento - determinar o conhecimento tácito, e coletar o conhecimento interno; f) desenho da estrutura tecnológica - considerar o conhecimento interno e externo; g) testar a tecnologia - executar testes da tecnologia; h) manutenção da tecnologia - conduzir reuniões para atualizações; i) re-teste - executar testes da tecnologia; j) formação para os trabalhadores - conduzir programas de formação sobre o uso das ferramentas de GC; I) apresentação do uso das práticas de GC - iniciar o uso da intranet; $\mathrm{m}$ ) rastrear o uso - criar relatórios de gestão; n) uso de sistemas - iniciar o uso de repositórios de dados externos.

Durante a GC devem ocorrer avaliações, considerando medição da qualidade e produtividade (refinar as técnicas), medição do desempenho das práticas de GC (rastrear o retorno do investimento) e condução de reuniões de 
avaliação das necessidades (comunicar os níveis de desempenho e melhoria contínua de oportunidades).

McCampbell, Clare e Gitters (1999) mencionam a coleta do conhecimento que pode ser relacionada à externalização, o treinamento que pode ser relacionado com a internalização, e a tecnologia necessária, que pode ser associado aos quatro modos de conversão. No entanto, isso é apresentado de um modo genérico, sem detalhar procedimentos ou indicar aspectos específicos para tratar o conhecimento tácito e o explícito.

\subsection{Soliman e Spooner (2000)}

Soliman e Spooner (2000) apresentam um conjunto de estratégias para a implementação da GC: alinhamento da GC com os objetivos de negócio; identificação dos benefícios da GC; escolha de um conjunto de atividades; implementação da estratégia de know-how; criação de ambiente de suporte à GC; adoção de tecnologias; criação de trabalho em equipe; e criação de uma liderança de GC. Para cada uma das estratégias citadas, os autores apresentam o papel da área de Recursos Humanos (RH). Por exemplo, para o alinhamento da GC com os objetivos de negócio, o papel do RH seria identificar onde o conhecimento tácito reside e como associá-lo à GC.

O framework proposto por Soliman e Spooner (2000) relaciona aspectos que afetam a conversão do conhecimento, sem tratar especificamente dos modos de conversão. Por exemplo, ao mencionar o trabalho em equipe, pode estar facilitando a socialização, já a adoção de tecnologias pode ser útil para os quatro modos de conversão do conhecimento, tanto como uma forma de aproximar indivíduos dispersos geograficamente quanto para permitir a documentação e o acesso ao conhecimento.

\subsection{Mentzas (2001)}

O framework apresentado por Mentzas (2001) é chamado de Know-Net. Esse framework integra a abordagem do conhecimento como produto (foco na documentação, dissociado das pessoas) e como processo (foco na partilha entre as pessoas). As principais características são: foco em acessar e organizar o conhecimento; conexão dos recursos, ou seja, a transferência do conhecimento ocorre das pessoas para os sistemas e também entre as pessoas; consideração de quatro níveis de interação (individual, equipe, organizacional e interorganizacional); e consideração de quatro elementos, que são estratégia, processo, estrutura e sistema (MENTZAS, 2001).

Os estágios desse framework são: 0 - desconhecimento sobre a GC; 1 planejamento, determinação da visão e do escopo do projeto de GC; 2 desenvolvimento, depois de elaborada a estratégia a ser adotada, considerando as pessoas, o processo e a tecnologia; 3 - operação, colocação em prática do projeto. Durante a realização de um projeto de GC deve ser considerada a medição do processo e resultados e, também, o treinamento dos funcionários em relação ao processo e às tecnologias adotadas.

A externalização e a combinação são abordadas indiretamente quando Mentzas (2001) menciona a transferência do conhecimento das pessoas para os 
sistemas, a socialização quando indica a transferência do conhecimento entre as pessoas e a internalização quando considera o treinamento. Em relação à consideração da tecnologia, pode-se associar aos quatro modos de conversão do conhecimento

\subsection{Ndlela e Toit (2001)}

O framework proposto por Ndlela e Toit (2001) apresenta questões para a organização, uma sequência de passos e diretrizes a serem utilizados na implementação da GC. Inicialmente, a organização deve refletir sobre o seguinte conjunto de questões (NDLELA; TOIT, 2001): como criar valor com 0 conhecimento e como o conhecimento pode contribuir para diminuir o custo ou aumentar o lucro? Como explorar características raras através da GC? O que dificilmente pode ser imitado pela concorrência? Qual a relação da GC com as decisões e processos de negócio da organização? Depois de responder essas questões, para estabelecer um programa de GC deve-se: realizar uma análise interna da organização e análise do ambiente externo; formular uma estratégia; desenvolver um plano para a GC; e implementar e avaliar a GC.

As diretrizes apresentadas pelos autores são (NDLELA; TOIT, 2001, p.164):

garantir uma forte ligação com os objetivos de negócio; utilizar a visão da organização; definir um líder do conhecimento; cultivar uma cultura de criação e compartilhamento do conhecimento; incentivar o aprendizado contínuo; desenvolver uma infra-estrutura tecnológica; desenvolver um processo do conhecimento sistemático na organização.

No framework apresentado por Ndlela e Toit (2001), a associação com os modos de conversão do conhecimento pode ser identificada no impacto que a cultura e a tecnologia podem causar. Além disso, o aprendizado contínuo pode ser associado à internalização.

\subsection{Rubenstein-Montano et al. (2001)}

O framework proposto por Rubenstein-Montano et al. (2001) é composto por atividades e por fatores que afetam essas atividades. As recomendações dos autores são: associar a GC aos objetivos da organização; planejar as atividades antes da execução; reconhecer a cultura da organização; tratar como um processo evolutivo, considerando feedback e aprendizagem; tratar apropriadamente o conhecimento explícito e o tácito; considerar atividades como encontrar, verificar, armazenar, organizar, compartilhar e usar; identificar o propósito da GC; e definir a tecnologia. Segundo os autores, a ordem das atividades em um framework não é relevante, pois elas acontecem ciclicamente.

Assim como outros frameworks, a tecnologia é mencionada por RubensteinMontano et al. (2001), o que impacta nos modos de conversão: socialização aproximar indivíduos dispersos geograficamente; e externalização e combinação facilitar a documentação; internalização - permitir o acesso ao conhecimento com 
a possibilidade de gerar novo conhecimento tácito. Além disso, o framework menciona tratar o conhecimento tácito e explícito, embora não diga como fazê-lo.

\subsection{Holsapple e Joshi (2002)}

O framework apresentado por Holsapple e Joshi (2002) é formado por três componentes considerados fundamentais para o processo de GC e suas relações. Esses componentes são: descrição dos recursos de conhecimento da organização; atividades para lidar com os recursos de conhecimento da organização; e tipos de influências na condução da GC.

Os recursos internos de uma organização (humano, material, financeiro, e conhecimento) assim como os recursos externos afetam uns aos outros. 0 conhecimento pode ser identificado em 6 formas: conhecimento dos participantes; cultura; infraestrutura; artefatos; propósito; estratégia. 0 conhecimento do ambiente não pertence à organização e também não pode ser controlado por ela. No entanto, pode ser uma fonte de conhecimento potencial para a organização.

O segundo componente do framework diz respeito às atividades para lidar com os recursos de conhecimento da organização, que são: aquisição; seleção; internalização; e utilização.

O terceiro componente desse framework visa auxiliar no reconhecimento de fatores que afetam a condução da GC na organização. Esses fatores podem ser de três tipos: recursos (financeiros, humanos, conhecimento e material), gerenciais (liderança, coordenação e avaliação) e ambientais (competidores, fornecedores, clientes e governo).

Segundo os autores, o principal objetivo desse framework é a generalização, ou seja, foi desenvolvido para ser aplicável em qualquer tipo de organização.

Esse framework não menciona, diretamente, aos modos de conversão do conhecimento. No entanto, os aspectos cultura e infraestrutura impactam nos modos de conversão.

\subsection{Jarrar (2002)}

O framework apresentado por Jarrar (2002) considera 4 elementos como os principais para a implementação da GC: a) definição da GC como prioridade estratégica e obtenção do comprometimento dos gestores; b) definição e entendimento do conhecimento organizacional; c) GC, considerando as melhores práticas em termos de infraestrutura e ferramentas; e d) ambiente do conhecimento, abrangendo aspectos como a cultura da organização e o desenvolvimento e criação do conhecimento. O autor apresenta, também, práticas e procedimentos a serem adotados para operacionalizar os elementos propostos.

O ponto inicial do framework proposto por Jarrar (2002) é o estabelecimento de prioridade estratégica para a GC. Isso é obtido, considerandose três aspectos: 1) alinhamento dos objetivos da GC com os objetivos do negócio, obtido pela definição do conhecimento e seu papel no negócio, identificação de lacunas nos recursos de conhecimento dos fornecedores e 
competidores continuamente, promovendo o desenvolvimento do capital intelectual; 2) associar a GC à criação de valor, definindo claramente os objetivos; e 3) suporte da alta administração, moral e financeiro.

Segundo Jarrar (2002), a definição e o entendimento do conhecimento organizacional são obtidos através do mapeamento do conhecimento, identificando como e onde o conhecimento é desenvolvido na organização, qual o seu fluxo e também quem são as pessoas que possuem conhecimento sobre determinados tópicos.

Em relação às ferramentas e infraestrutura, Jarrar (2002) considera: criação de múltiplos canais de acesso ao conhecimento; criação de repositórios para o conhecimento externo, interno e interno informal; adoção de tecnologia da informação que atenda às necessidades da organização; definição de uma equipe para coordenar o processo de GC; adoção de métricas para avaliar o capital intelectual e a GC.

O ambiente do conhecimento engloba a cultura da organização e a criação e desenvolvimento do conhecimento (JARRAR, 2002). Para estabelecer uma cultura propícia à GC, o autor sugere algumas práticas como, por exemplo, estimular os funcionários a partilharem o conhecimento, e associar esta partilha com o sistema de recompensa da organização. Para a criação e desenvolvimento do conhecimento o autor sugere práticas como recrutamento das melhores pessoas, desenvolvimento dos funcionários (formação, mentoring, etc.), e aumento contínuo dos desafios dos funcionários.

No framework apresentado por Jarrar (2002) é possível identificar aspectos (por exemplo, cultura, tecnologia, entre outros) que impactam nos quatros modos de conversão do conhecimento. Além disto, a socialização é tratada quando no framework é mencionada a identificação de quem são as pessoas que possuem conhecimento sobre determinados tópicos; a externalização e a combinação estão relacionadas com o mapeamento do conhecimento e também com a existência de um repositório para o conhecimento; e a internalização com o acesso ao repositório do conhecimento, podendo gerar novos conhecimentos tácitos.

\subsection{Lai e Chu (2002)}

O framework apresentado por Lai e Chu (2002) considera três aspectos, que são recursos do conhecimento, atividades de GC e influências na GC.

Os três tipos de recursos do conhecimento são humano, relacionado com a capacidade de resolver problemas; estrutural, que diz respeito à capacidade da organização em atender às necessidades do mercado; e consumidor, representando a relação entre a organização e os seus stakeholders (LAI; CHU, 2002).

As cinco atividades de GC são: iniciação, que corresponde à preparação da organização para a GC; geração, que diz respeito ao mapeamento do conhecimento existente na organização e criação de novos conhecimentos; modelagem, que representa a organização do conhecimento para o armazenamento; repositório, que considera o local para armazenamento do conhecimento, visando a sua fácil recuperação pelos usuários; distribuição e transferência, que corresponde à forma como o conhecimento é distribuído para as pessoas; utilização, que diz respeito à aplicação do conhecimento nos 
processos da organização; retrospectiva, que representa a avaliação do processo (LAI; CHU, 2002).

Os fatores considerados facilitadores ou inibidores da GC são: cultura, liderança, avaliação, educação, sistema de incentivo, capacidade de adaptação da organização, valores e normas, tecnologia (LAI; CHU, 2002).

Além dos aspectos (por exemplo, cultura, liderança, tecnologia, etc.) que podem impactar nos quatro modos de conversão do conhecimento, a externalização e a combinação podem ser indiretamente identificadas com o mapeamento do conhecimento existente e o repositório para armazenamento do conhecimento; já a internalização está relacionada com a recuperação do conhecimento, aplicação nos processos da organização e também com a criação de novos conhecimentos.

\subsection{Wong e Aspinwall (2004)}

Wong e Aspinwall (2004) analisaram um conjunto de frameworks e propuseram diretrizes para o desenvolvimento de um framework para a implementação da GC.

As diretrizes propostas são (WONG; ASPINWALL, 2004): adotar uma estrutura clara, independentemente das atividades a serem realizadas; considerar os diferentes tipos de conhecimento, explícito e tácito, pois cada um demanda diferentes atividades; incluir os processos como, criação, armazenamento, disseminação e uso; identificar os fatores que influenciam a GC como, por exemplo, a cultura; e garantir um equilíbrio entre a abordagem tecnológica e social.

Os modos de conversão do conhecimento são identificados apenas indiretamente no framework proposto por Wong e Aspinwall (2004). Isso ocorre quando os autores mencionam a necessidade de considerar o conhecimento tácito, o explícito e, também, a cultura.

\subsection{Lin (2007)}

Lin (2007) apresenta a GC através de um contínuo de três estágios. O primeiro estágio, chamado iniciação, onde a organização reconhece a importância da GC e se prepara para a implementação. O segundo estágio, denominado de desenvolvimento, a organização considera a infraestrutura para facilitar e motivar as atividades da GC. O último estágio, definido como maturidade, a GC considera o ambiente interno e o externo.

No primeiro estágio, denominado de iniciação, a organização define e dissemina entre os funcionários os objetivos da GC e os critérios para avaliação da utilidade do conhecimento (LIN, 2007). Nesse estágio é definida uma equipe para dar início à GC. No entanto, as atividades de GC são limitadas, pois a infraestrutura ainda não está totalmente implementada. No desenvolvimento, segundo estágio, a organização investe na infraestrutura de GC (LIN, 2007). Isso inclui a definição de um líder para a GC, aspectos culturais, estrutura organizacional e políticas de RH. O último estágio ocorre quando a GC considera o ambiente interno da organização, assim como os fornecedores e clientes (LIN, 
2007). Nesse estágio é que a melhoria do desempenho organizacional pode ser observada.

Nesse framework se percebe aspectos (por exemplo, cultura e infraestrutura) que podem impactar nos modos de conversão do conhecimento. No entanto, diretamente, nenhum dos modos de conversão é abordado pelo framework proposto por Lin (2007).

\subsection{Mehta (2008)}

Mehta (2008) apresenta um framework para implementação da GC em três estágios. O primeiro estágio corresponde à articulação sobre a intenção estratégica da GC. Nesse estágio, são definidos a visão do programa de GC e os objetivos e, por isso, é possível identificar as lacunas do conhecimento na organização. O segundo estágio diz respeito à facilitação do fluxo do conhecimento com vistas à inovação. Nesse estágio deve ser considerada a infraestrutura humana e tecnológica. O terceiro estágio está relacionado com a avaliação do valor da GC, na qual são desenvolvidas medidas diretas e indiretas para essa avaliação.

No framework proposto por Mehta (2008) os modos de conversão do conhecimento podem ser identificados indiretamente, apenas através da indicação da necessidade de definir a tecnologia.

\subsection{Frameworks de implementação de GC e a espiral do conhecimento}

O nível de detalhamento dos frameworks apresentados é diferente. Por exemplo, Ndlela e Toit (2001) apresentam uma sequência de passos a serem percorridos para a implementação da GC, sendo que, para alguns deles, sugerem inclusive a forma de executar. Já Wong e Aspinwall (2004), colocam as diretrizes sem indicação do modo de execução das mesmas, ou seja, o framework possui um nível de informação mais agregado. Os principais aspectos mencionados pelos frameworks são relacionados no QUADRO 1.

QUADRO 1

Aspectos mencionados nos frameworks 


\begin{tabular}{|c|c|c|c|c|c|c|c|c|c|c|c|c|c|c|}
\hline $\begin{array}{l}\text { Aspectos mencionados nos } \\
\text { frameworks }\end{array}$ & 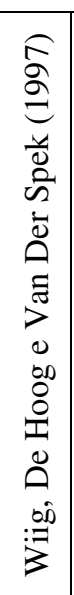 & 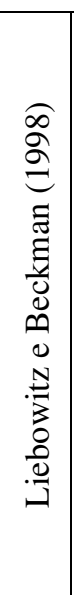 & 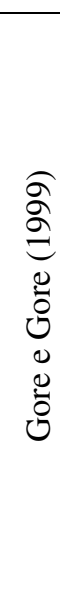 & 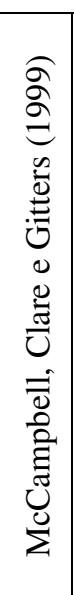 & 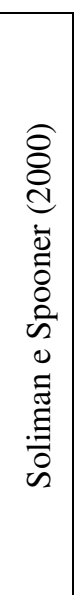 & 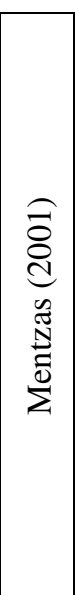 & 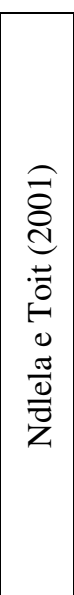 & 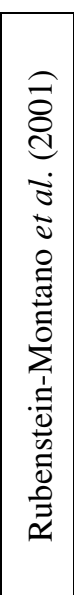 & 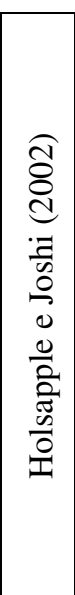 & 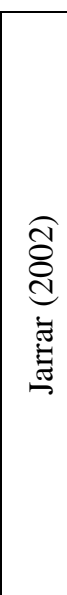 & 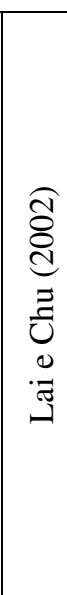 & 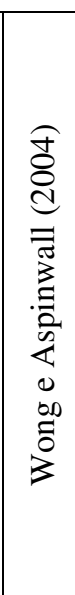 & $\begin{array}{l}\underset{\mathfrak{8}}{8} \\
\underset{]}{\Xi}\end{array}$ & 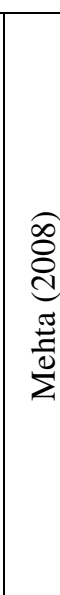 \\
\hline $\begin{array}{l}\text { Alinhamento com os objetivos de } \\
\text { negócio }\end{array}$ & & & & & $\mathrm{X}$ & & $\mathrm{X}$ & $\mathrm{X}$ & & $\mathrm{X}$ & & & & \\
\hline Ambiente externo & & & & & & & & & $\mathrm{X}$ & $\mathrm{X}$ & & & $\mathrm{X}$ & \\
\hline Avaliação da GC & $\mathrm{X}$ & & & $\mathrm{X}$ & & $\mathrm{X}$ & $\mathrm{X}$ & $\mathrm{X}$ & $\mathrm{X}$ & $\mathrm{X}$ & $\mathrm{X}$ & & & $\mathrm{X}$ \\
\hline Benefícios & & & & & $\mathrm{X}$ & & & & & & $\mathrm{X}$ & & & \\
\hline Comunicação & & & & $\mathrm{X}$ & & & & & & & & & & \\
\hline Conhecimento explícito e tácito & & & $\mathrm{X}$ & $\mathrm{X}$ & & $\mathrm{X}$ & & $\mathrm{X}$ & & $\mathrm{X}$ & & $\mathrm{X}$ & & \\
\hline Cultura & & $\mathrm{X}$ & & & $\mathrm{X}$ & & $\mathrm{X}$ & $\mathrm{X}$ & $\mathrm{X}$ & $\mathrm{X}$ & $\mathrm{X}$ & $\mathrm{X}$ & $\mathrm{X}$ & \\
\hline Estratégia & & $\mathrm{X}$ & & & & $\mathrm{X}$ & $\mathrm{X}$ & & $\mathrm{X}$ & & & & & \\
\hline Fases do processo de GC & $\mathrm{X}$ & $\mathrm{X}$ & $\mathrm{X}$ & & $\mathrm{X}$ & $\mathrm{X}$ & $\mathrm{X}$ & $\mathrm{X}$ & $\mathrm{X}$ & & $\mathrm{X}$ & $\mathrm{X}$ & & \\
\hline Mapeamento do conhecimento & $\mathrm{X}$ & $\mathrm{X}$ & $\mathrm{X}$ & $\mathrm{X}$ & & & & & $\mathrm{X}$ & $\mathrm{X}$ & $\mathrm{X}$ & & & $\mathrm{X}$ \\
\hline Objetivos da GC & & & & & & $\mathrm{X}$ & & $\mathrm{X}$ & $\mathrm{X}$ & $\mathrm{X}$ & & & $\mathrm{X}$ & $\mathrm{X}$ \\
\hline Plano de melhorias & $\mathrm{X}$ & & & & & & $\mathrm{X}$ & $\mathrm{X}$ & & & & $\mathrm{X}$ & & \\
\hline Recursos financeiros & & & & & & & & & $\mathrm{X}$ & & & & & \\
\hline Responsável pela GC e equipe & & $\mathrm{X}$ & & $\mathrm{X}$ & $\mathrm{X}$ & & $\mathrm{X}$ & & $\mathrm{X}$ & $\mathrm{X}$ & & & $\mathrm{X}$ & \\
\hline $\begin{array}{l}\text { Sistema para motivar a } \\
\text { participação }\end{array}$ & & $\mathrm{X}$ & & & & & & & & $\mathrm{X}$ & $\mathrm{X}$ & & $\mathrm{X}$ & \\
\hline Suporte da alta administração & & $\mathrm{X}$ & $\mathrm{X}$ & $\mathrm{X}$ & & & & & $\mathrm{X}$ & $\mathrm{X}$ & $\mathrm{X}$ & & $\mathrm{X}$ & \\
\hline Tecnologia & & $\mathrm{X}$ & & $\mathrm{X}$ & $\mathrm{X}$ & $\mathrm{X}$ & $\mathrm{X}$ & $\mathrm{X}$ & $\mathrm{X}$ & $\mathrm{X}$ & $\mathrm{X}$ & $\mathrm{X}$ & $X$ & $\mathrm{X}$ \\
\hline Treinamento & & & & $\mathrm{X}$ & & $\mathrm{X}$ & $\mathrm{X}$ & & & & & & & \\
\hline
\end{tabular}

Fonte: Dados da pesquisa.

A variação no grau de detalhamento também ocorre em relação à abordagem da espiral do conhecimento nos frameworks. Por exemplo, o framework apresentado por Lin (2007) indica a necessidade de definir modos de conversão do conhecimento, Wong e Aspinwall (2004) mencionam a necessidade de considerar os diferentes tipos de conhecimento (tácito e explícito) e que isso requer diferentes atividades. Já Soliman e Spooner (2000) citam atividades que favorecem a socialização, como a realização de trabalho em equipe.

A relação dos frameworks com a espiral do conhecimento é apresentada no QUADRO 2. Neste Quadro é indicado o termo "Atividade", caso alguma prática no framework possua relação com o modo de conversão do conhecimento e "Elemento", caso algum aspecto possa impactar positiva ou negativamente nos modos de conversão do conhecimento.

\section{QUADRO 2}

Frameworks e a espiral do conhecimento 


\begin{tabular}{|l|l|l|l|l|}
\hline Wiig, De Hoog e Van Der Spek (1997) & Atividade & Atividade & Atividade & Atividade \\
\hline Liebowitz e Beckman (1998) & Elemento & $\begin{array}{l}\text { Atividade } \\
\text { Elemento }\end{array}$ & $\begin{array}{l}\text { Atividade } \\
\text { Elemento }\end{array}$ & $\begin{array}{l}\text { Atividade } \\
\text { Elemento }\end{array}$ \\
\hline Gore e Gore (1999) & Atividade & Atividade & Atividade & Atividade \\
\hline McCampbell, Clare e Gitters (1999) & & Atividade & & $\begin{array}{l}\text { Atividade } \\
\text { Elemento }\end{array}$ \\
\hline Soliman e Spooner (2000) & Elemento & Elemento & Elemento & \\
& Elividade & & & \\
& Elemento & Elemento & Elemento & Elemento \\
\hline Mentzas (2001) & Atividade & Atividade & Atividade & Atividade \\
& Elemento & Elemento & Elemento & Elemento \\
\hline Ndlela e Toit (2001) & & & & Atividade \\
& Elemento & Elemento & Elemento & Elemento \\
\hline Rubenstein-Montano et al. (2001) & Elemento & Elemento & Elemento & Elemento \\
\hline Holsapple e Joshi (2002) & Elemento & Elemento & Elemento & Elemento \\
\hline Jarrar (2002) & Atividade & Atividade & Atividade & Atividade \\
& Elemento & Elemento & Elemento & Elemento \\
\hline Lai e Chu (2002) & & Atividade & Atividade & Atividade \\
& Elemento & Elemento & Elemento & Elemento \\
\hline Wong e Aspinwall (2004) & Elemento & Elemento & Elemento & Elemento \\
\hline Lin (2007) & Elemento & Elemento & Elemento & Elemento \\
\hline Mehta (2008) & Elemento & Elemento & Elemento & Elemento \\
\hline
\end{tabular}

Fonte: Dados da pesquisa.

Ao analisar o QUADRO 2, é possível perceber que a maioria dos frameworks indica elementos que podem afetar os modos de conversão do conhecimento como, por exemplo, a tecnologia. No entanto, apenas cerca da metade deles possui atividades que podem ser associadas aos modos de conversão do conhecimento, como por exemplo, a adoção de lições aprendidas.

\section{Método}

Este trabalho é exploratório por natureza. A partir do objetivo da pesquisa, a estratégia definida foi o estudo de caso múltiplo. O estudo de caso permite analisar o fenômeno no seu ambiente natural, podendo utilizar múltiplos métodos de coleta de dados para obter informação de uma ou várias entidades (pessoas, grupos ou organizações) (YIN, 2005). Optou-se por um estudo de caso múltiplo em duas organizações, uma vez que as conclusões obtidas através de dois casos são mais contundentes do que conclusões obtidas através de apenas um caso único (YIN, 2005).

Nesta pesquisa, foram selecionadas duas organizações operando em Portugal, na área de tecnologia da informação, considerando como principal critério de escolha a existência de GC. Por questões de sigilo, as organizações serão chamadas de A (capital majoritariamente português com cerca de 400 funcionários) e $\mathrm{B}$ (multinacional com cerca de 400 funcionários em Portugal).

Como estratégia para aumentar a confiabilidade da pesquisa, um protocolo foi desenvolvido, com a visão geral do estudo de caso, procedimentos, instrumento de coleta de dados e o guia para o relatório do estudo de caso.

Esta pesquisa foi constituída de duas etapas. Na primeira foram identificados os frameworks e realizada a análise de conteúdo dos mesmos, à luz dos quatro modos de conversão do conhecimento (socialização, externalização, combinação e internalização), com o objetivo de verificar como os frameworks de implementação de GC abordam a conversão do conhecimento. Na segunda etapa 
desta pesquisa, foram realizadas duas entrevistas em cada organização, com o intuito de verificar como a conversão do conhecimento está sendo contemplada nessas organizações. Os entrevistados são 2 gestores de GC e 2 participantes do projeto de GC. As entrevistas realizadas, nesta pesquisa, foram semiestruturadas, realizadas presencialmente por um dos autores, com duração aproximada de $1 \mathrm{~h}$ $30 \mathrm{~min}$ cada uma. O roteiro de pesquisa, considerando os aspectos identificados pelos frameworks de implementação da GC (QUADRO 1) e os modos de conversão do conhecimento (seção 2.1), foi submetido para validação de dois professores com atuação na pós-graduação. Os ajustes solicitados foram realizados. Todas as entrevistas foram gravadas e transcritas.

A análise de conteúdo temática foi a técnica adotada para análise dos dados coletados através das entrevistas. As categorias consideradas foram os aspectos identificados pelos frameworks de implementação da GC (QUADRO 1) e os quatro modos de conversão do conhecimento.

\section{Gestão do Conhecimento nas organizações A e B} (4.2).

Esta seção apresenta uma visão geral da GC nas organizações A (4.1) e B

\subsection{GC na organização A}

A GC na organização A está sob a responsabilidade da área de recursos humanos. Em 2001, foi implantado o sistema da qualidade na organização e também criada a knowledge base, sendo a inspiração obtida na metodologia de gestão de projetos. Sempre existiu a preocupação de que os funcionários não empregassem mais tempo fazendo a documentação do que fazendo o projeto. Na implementação da GC a organização não utilizou um framework específico, mas aproxima-se do framework apresentado por Jarrar (2002), considerando os aspectos contemplados pela organização em relação à GC. Nessa empresa, é possível identificar a presença dos seguintes aspectos: alinhamento com os objetivos de negócio; avaliação da GC; conhecimento explícito e tácito; cultura; fases do processo; objetivo da GC; responsável pela GC e equipe; suporte da alta administração; tecnologia; e treinamento. Entre eles, estão 6 dos 7 aspectos contemplados pela maioria dos frameworks, sendo que apenas o mapeamento do conhecimento não foi identificado.

O objetivo da GC, nessa empresa, é possuir uma imagem única perante o cliente, independente do país que esteja atuando, o qual está alinhado com os objetivos de negócio da organização, segundo os entrevistados. Através das entrevistas, foi possível verificar que existe o suporte da alta administração, o que, para os funcionários, ainda não é um processo totalmente incorporado ao dia a dia, exigindo que a alta administração reforce a necessidade da realização das atividades de GC e, também, que exista uma auditoria interna para verificar a execução dessas atividades. Isso reforça a importância da cultura para a GC, que é um dos elementos citados pelos frameworks descritos anteriormente.

Nessa organização existe uma preocupação com o armazenamento dos documentos, assim como, com a disponibilização dos currículos dos funcionários. 
Isso demonstra uma preocupação consolidada em relação ao conhecimento explícito e um movimento inicial quanto ao conhecimento tácito.

O acesso ao conteúdo armazenado é determinado pelo perfil do funcionário, embora seja característica dessa organização a transparência, ou seja, a maioria do conteúdo está disponível para a maioria das pessoas. O conteúdo armazenado é previamente aprovado por uma comissão executiva. A estrutura definida para o armazenamento do conteúdo já foi reformulada, no intuito de reduzir o tempo alocado para os procedimentos de armazenamento. Os documentos são armazenados em um local único, com a possibilidade de acesso remoto, o que é importante, considerando a atuação internacional da organização. A tecnologia, nesse caso, é considerada importante, em função da necessidade de acesso remoto, do volume de documentos e da possibilidade de busca de conteúdo através de determinados atributos. A organização possui um portal.

Os entrevistados acreditam que a auditoria é um fator fundamental para que os funcionários armazenem os documentos. Existe um processo de monitoramento através das auditorias internas, sendo divulgado o nome daqueles que tiverem alguma não conformidade em relação ao processo. No entanto, não existe uma sistemática de recompensa para aqueles que não apresentarem não conformidades. Na percepção dos entrevistados, ainda é necessário melhorar a partilha de questões técnicas, ou seja, o aprendizado em relação ao processo e não somente à documentação dos projetos. A organização também possui uma preocupação com a formação dos colaboradores em relação à GC e, também, quanto a conteúdos técnicos e de gestão.

\subsection{GC na organização B}

Informalmente a GC sempre existiu na organização B, a partir de 2006, quando os procedimentos começaram a ser sistematizados. Na implementação da GC, a organização B também não utilizou um framework específico, mas, aproximou-se do framework apresentado por Jarrar (2002). Nessa empresa, é possível identificar a presença dos seguintes aspectos: alinhamento com os objetivos de negócio; ambiente externo; avaliação da GC; comunicação; conhecimento explícito e tácito; cultura; fases do processo; mapeamento do conhecimento; objetivo da GC; responsável pela GC e equipe; sistema para motivar a participação; suporte da alta administração; tecnologia; e treinamento. Entre os 14 aspectos identificados na organização B estão os 7 contemplados pela maioria dos frameworks analisados.

O objetivo da GC na organização B é o aumento da produtividade, o qual está alinhado com os objetivos de negócio. Nessa organização, a GC possui o suporte da alta administração desde o início, sendo que a cultura da partilha foi desenvolvida na organização.

$\mathrm{Na}$ organização $\mathrm{B}, \mathrm{a}$ GC está sob a coordenação da área de recursos humanos, mas, sendo desenvolvida por um grupo de pessoas oriundas de cada uma das áreas da organização. Essa atribuição de responsabilidades aos funcionários das diferentes áreas da organização foi considerada fundamental para o comprometimento de todos com a GC, na organização. Esse grupo foi responsável por identificar como a partilha ocorria, onde estavam os problemas e quais ferramentas estavam sendo utilizadas. A partir desse mapeamento, foram 
selecionadas as ferramentas mais adequadas, o conteúdo a ser armazenado, a estruturação das comunidades de conhecimento, entre outras práticas de GC.

O acesso ao conteúdo armazenado é diferenciado, segundo o perfil do funcionário. As práticas de GC adotadas pela organização são: comunidades de conhecimento; blog; Intranet; repositório; programa para suportar a saída do funcionário; reuniões com parceiros e clientes; reuniões de lições aprendidas; etc. Essas práticas permitem identificar uma preocupação com o conhecimento tácito e explícito. Nessa organização já existe um envolvimento de clientes e parceiros na GC, através, por exemplo, de reuniões para discussão de produtos.

A organização possui uma sistemática de premiação anual associada à GC. Os funcionários participam de treinamento sobre as práticas utilizadas para a GC. A tecnologia de informação utilizada para a GC já existia na organização, tendo sido necessário apenas padronizar a utilização das ferramentas para cada função e a realização de customizações. Anualmente a GC é avaliada pelos funcionários qualitativamente. Até o momento, não existe nenhum indicador que associe a GC aos resultados da organização.

\section{Conversão do conhecimento nos frameworks e nas organizações}

Na sequência, é discutida a relação entre os modos de conversão do conhecimento e os frameworks de GC (5.1 a 5.4).

\subsection{Socialização - tácito para tácito}

As atividades relacionadas com a socialização nos frameworks de Wiig, De Hoog e Van Der Spek (1997), Gore e Gore (1999), Soliman e Spooner (2000), Mentzas (2001) e Jarrar (2002) identificadas foram: identificação das pessoas com conhecimento em determinado tópico, lições aprendidas, trabalho em equipe e comunidades de prática.

Nas duas organizações, identificou-se a preocupação com o compartilhamento do conhecimento tácito, embora isso seja mais visível na organização B. Nas organizações A e B, é possível obter na intranet o currículo dos funcionários, identificando a experiência de cada um e formas de contato. A busca dos currículos é realizada pelo nome dos funcionários, o que pode ser aprimorado, permitindo identificar os funcionários com determinada competência. Em ambas as organizações, as reuniões de lições aprendidas encorajam os funcionários a discutirem os problemas enfrentados abertamente. O trabalho em equipe também ocorre nas duas organizações. Em relação à facilitação de reuniões sociais entre os funcionários, percebe-se ser uma preocupação apenas na organização $\mathrm{B}$, onde existe a prática de comunidades de conhecimento, happy hour, reuniões informais entre os funcionários e também reuniões com clientes e parceiros.

Com exceção dos frameworks de Wiig, De Hoog e Van Der Spek (1997) e Gore e Gore (1999), os demais apontam a necessidade de adotar tecnologia facilitadora da GC, a qual possa colocar as pessoas em contato. Nas duas organizações pesquisadas, verificou-se a adoção de tecnologias que suportam a GC (com funcionários trabalhando no escritório do cliente), o que certamente é 
facilitado pelo fato de atuarem na área de tecnologia de informação. Uma preocupação com a cultura, com foco na partilha, também foi identificada nas duas organizações e mencionada em 9 frameworks, como, por exemplo, em Liebowitz e Beckman (1998 apud LIEBOWITZ, 1999), o que facilita o compartilhamento do conhecimento entre os colaboradores.

\subsection{Externalização - tácito para explícito}

As atividades relacionados com a externalização nos frameworks de Wiig, De Hoog e Van Der Spek (1997), Liebowitz e Beckman (1998), Gore e Gore (1999), McCampbell, Clare e Gitters (1999), Mentzas (2001), Jarrar (2002), Lai e Chu (2002) identificadas foram: documentação de lições aprendidas, documentação do conhecimento compartilhado nas comunidades de prática, mapeamento do conhecimento, captura e armazenamento do conhecimento em repositórios.

Nas duas organizações, identificou-se atenção com a transformação do conhecimento tácito em explícito, sendo essa a ênfase da organização A. Na organização B foi realizado o mapeamento do conhecimento existente, das tecnologias adotadas e das necessidades e problemas enfrentados pelos funcionários, para que a partir disso, fossem definidas as diretrizes da GC.

O armazenamento do conhecimento é a preocupação central na organização A, onde existe a realização de auditoria interna para garantir que o conhecimento seja documentado e armazenado periodicamente em um único repositório. Na organização B, o conhecimento também é armazenado, mas existem repositórios acessados a partir da intranet que são próprios para cada uma das áreas da organização. Em ambas as organizações, as reuniões de lições aprendidas contribuem para a externalização do conhecimento.

Uma forma diferenciada identificada na organização B é o story telling, onde casos de sucesso ou insucesso são documentados através de uma história contada com a utilização de personagens. Os funcionários receberam treinamento sobre como montar essas histórias e o relato das histórias é transformado em vídeos.

Outros aspectos que indiretamente podem ser associados à externalização, identificados nos frameworks foram: encorajar os funcionários a discutirem seus erros abertamente e a documentar os mesmos; adotar tecnologia facilitadora de GC; e adotar um programa de lições aprendidas. Esses aspectos foram identificados em ambas as organizações.

A organização B possui um programa para o caso da saída de um funcionário da organização, que pode ser associado à externalização. Nesse caso, um repositório é criado para armazenar o conhecimento relacionado a essa pessoa (comunidades de partilha que tinha participação, relatórios dos projetos em que estava trabalhando, etc.).

\subsection{Combinação - explícito para explícito}


As práticas mencionadas nos frameworks que podem ser associadas à combinação são: lições aprendidas geradas por diferentes grupos são combinadas, gerando novo conhecimento explícito, resolução de problemas de conhecimentos conflitantes, análise do conhecimento explícito existente, adoção de tecnologia facilitadora de GC. Esses aspectos podem ser identificados, por exemplo, em: Wiig, De Hoog e Van Der Spek (1997); Liebowitz e Beckman (1998 apud LIEBOWITZ, 1999); Gore e Gore (1999), entre outros.

A combinação pode ser identificada na organização $A$, através da análise dos documentos por parte de uma comissão executiva antes da sua publicação no repositório. Na organização $\mathrm{B}$, também existem pessoas responsáveis pela atualização dos repositórios. A combinação também pode ser realizada através do programa de lições aprendidas, existente nas duas organizações e na adoção de tecnologia facilitadora da GC. O programa de lições aprendidas também contribui nessas duas organizações para a conversão do conhecimento explícito em explícito.

\subsection{Internalização - explícito para tácito}

As atividades relacionadas com a internalização nos frameworks de Wiig, De Hoog e Van Der Spek (1997), Liebowitz e Beckman (1998 apud LIEBOWITZ, 1999), Gore e Gore (1999), McCampbell, Clare e Gitters (1999), Mentzas (2001), Ndlela e Toit (2001), Jarrar (2002) e Lai e Chu (2002) identificadas foram: acesso ao repositório, acesso às lições aprendidas, criação de novos produtos e serviços desenvolvidos com base no conhecimento e treinamento. As organizações $A$ e $B$ possuem repositórios que os funcionários podem ter acesso, lições aprendidas documentadas e treinamento. Não foi possível verificar a criação de novos produtos ou serviços a partir do acesso ao conhecimento nas organizações $A$ e $B$.

A distribuição do conhecimento auxilia na internalização. Nas organizações $A$ e $B$, os funcionários possuem acesso à base de conhecimento de qualquer local. Na organização $B$, existem diferentes perfis de acesso à base de conhecimento. $A$ necessidade de se adotar uma tecnologia facilitadora da GC, apontada por diversos frameworks, como mostra o Quadro 2, permite que o conhecimento seja acessado, possibilitando a geração de novos conhecimentos tácitos. A adoção de tecnologia é facilitada, nessas organizações, por ser a área de atuação delas.

A recuperação e utilização do conhecimento, gerando novo conhecimento tácito, ficam a critério dos funcionários, sendo um ponto frágil de ser observado, especialmente porque ainda não existem métricas que permitam verificar o resultado da GC nas organizações analisadas.

\section{Conclusão}

As contribuições desta pesquisa são tanto para a comunidade acadêmica quanto para os gestores de organizações. A primeira é a comparação dos frameworks à luz da espiral do conhecimento. A segunda, diz respeito às práticas que duas organizações, localizadas em Portugal, estão adotando em cada um dos modos de conversão do conhecimento.

O nível de detalhamento dos frameworks de implementação de GC é variável, sendo os frameworks propostos por Holsapple e Joshi (2002) e Jarrar (2002) os que tratam de um maior número de aspectos. Os aspectos identificados no maior número de frameworks foram: tecnologia; fases do processo; avaliação; 
cultura; mapeamento do conhecimento; responsável pela GC e equipe; e suporte da alta administração.

A implementação da GC se aproxima nas organizações A e B do framework de Jarrar (2002). Na organização A, não são considerados 3 aspectos mencionados pelo framework (ambiente externo, mapeamento do conhecimento, sistema para motivar a participação). Já a organização B tem uma preocupação com todos os aspectos considerados no framework de Jarrar (2002), com destaque para a comunicação, fases do processo e treinamento. Os aspectos contemplados pelas organizações podem estar relacionados com as características delas e também com a experiência de cada uma em relação à GC.

A GC supõe, por definição, a inclusão do conhecimento interno e externo. No entanto, apenas 3 frameworks, que são Holsapple e Joshi (2002), Jarrar (2002) e Lin (2007) contemplam o ambiente externo explicitamente como parte de seus princípios norteadores para implementação da GC. A organização B relata que a participação dos clientes e fornecedores só pode ocorrer depois que a GC estiver organizada internamente.

Os 14 frameworks de GC analisados apresentam princípios norteadores para a implementação. No entanto, eles não abordam em profundidade os modos de conversão. Seis deles colocam a necessidade de contemplar o conhecimento tácito e o explícito, mas sem descrever como fazer isso. Os frameworks atribuem maior atenção aos aspectos relacionados com as condições para a implementação da GC (apoio da alta administração, cultura, sistema para motivar os participantes, etc.), do que propriamente com a criação do conhecimento (modos de conversão do conhecimento). Talvez isso possa ser explicado pelo objetivo desses frameworks, que é apresentar diretrizes e não a forma de operacionalização.

A organização A possui um foco maior na documentação do conhecimento, ou seja, com a passagem do conhecimento tácito para o explícito (externalização). Nessa organização, percebe-se uma maior preocupação com as condições para a implementação da GC. Já a organização B foca nos dois tipos de conhecimento e, portanto, maior ênfase nos quatro modos de conversão do conhecimento, adotando diferentes práticas para a conversão do conhecimento. Essa diferença entre as organizações talvez possa ser explicada pelo fato de que a importância da GC e a cultura da partilha já estejam incorporadas no dia a dia da organização $B$ e, ainda, necessitem ser desenvolvidas na organização $A$.

Em relação à socialização, identificaram-se reuniões de lições aprendidas; adoção de tecnologias de suporte; a interação entre indivíduos; disponibilizar caminhos para a identificação de especialistas (currículos); comunidades de prática; e reuniões com clientes e parceiros. Quanto à externalização, as práticas identificadas foram: documentação de lições aprendidas; adoção de tecnologias de suporte ao armazenamento; mapeamento do conhecimento; armazenamento do conhecimento; story telling; e programa para a saída de funcionários. Para a combinação, as práticas evidenciadas foram: resolução de conhecimentos conflitantes; lições aprendidas de diferentes grupos; adotar tecnologias que suportem o armazenamento e a busca do conhecimento; armazenamento do conhecimento; e análise dos documentos antes da publicação nos repositórios. Em termos de internalização, as práticas consideradas foram: a recuperação e uso do conhecimento e o treinamento. 
Esta pesquisa não aprofundou a análise sobre as práticas de GC que contribuem para a conversão do conhecimento, o que pode ser considerada uma limitação. Como pesquisa futura, pretende-se investigar as práticas de GC que favoreçam a conversão do conhecimento, assim como, desenvolver um framework para implementação da GC, que considere os modos de conversão do conhecimento, além das condições necessárias para o alcance dos objetivos da GC.

\section{Referências}

GORE, C.; GORE, E. Knowledge management: the way forward. Total Quality Management, v. 10, n. 4-5, p. 554-560, 1999.

HOLSAPPLE, C. W.; JOSHI, K. D. Knowledge management: a threefold framework. The Information Society, v. 18, n. 1, p. 47-64, 2002.

ISHIKURA, Y. Gestão do conhecimento e concorrência global: a abordagem da Olympus à gestão do conhecimento global na indústria de câmeras fotográficas digitais. In: TAKEUCHI, H.; NONAKA, I. Gestão do conhecimento. Porto Alegre: Bookman, 2008. p.165-200.

JARRAR, Y. F. Knowledge management: learning for organizational experience. Managerial Auditing Journal, v. 17, n. 6, p. 322-328, 2002.

LAI, H. ; CHU, T. Knowledge management: a review of industrial cases. Journal of Computer Information Systems, v. 42, n. 5, p. 26-39, 2002.

LIEBOWITZ, J.; BECKMAN, T. Knowledge organizations: what every manager should know. Boca Raton: St. Lucie/CRC Press, 1998 apud LIEBOWITZ, J. Key ingredients to the success of an organization's knowledge management strategy. Knowledge and Process Management, v. 6, n. 1, p. 37-40, 1999.

LIEBOWITZ, J. Key ingredients to the success of an organization's knowledge management strategy. Knowledge and Process Management, v. 6, n. 1, p. 37-40, 1999.

LIN, H. A stage model of knowledge management: an empirical investigation of process and effectiveness. Journal of Information Science, v. 33, n. 6, p. 643659, 2007.

MCCAMPBELL, A. S.; CLARE, L. M.; GITTERS, S. H. Knowledge management: the new challenge for the 21st century. Journal of Knowledge Management, v. 3, n. 3, p. 172-179, 1999.

MEHTA, N. Successful knowledge management implementation in global software companies. Journal of Knowledge Management, v. 12, n. 2, p. 42-56, 2008.

MENTZAS, G. An holistic approach to realizing the full value of your "knowledge assets". Knowledge Management Review, v. 4, n. 3, p. 10-11, 2001.

NDLELA, L. T.; TOIT, A. S. Establishing a knowledge management programme for competitive advantage in an enterprise. International Journal of Information Management, v. 21, n. 2, p. 151-165, 2001. 
NONAKA, I. A dynamic theory of organizational knowledge creation. Organization Science, v.5, n.1, p. 14-37, 1994.

NONAKA; I.; TAKEUCHI, H. Teoria da criação do conhecimento organizacional. In: TAKEUCHI, H.; NONAKA, I. Gestão do conhecimento. Porto Alegre: Bookman, 2008. p.54-90.

RUBENSTEIN-MONTANO, B. et al. A systems thinking Framework for knowledge management. Decision Support Systems, v. 31, n. 1, p. 5-16, 2001.

SOLIMAN, F.; SPOONER, K. Strategies for implementing knowledge management: role of human resources management. Journal of Knowledge Management, v. 4, n. 4, p. 337-345, 2000.

TAKEUCHI, H.; NONAKA, I. Criação e dialética do conhecimento. In: TAKEUCHI, H. ; NONAKA, I. Gestão do conhecimento. Porto Alegre: Bookman, 2008. p. 17-38. WIIG, K. M.; DE HOOG, R.; VAN DER SPEK, R. Supporting knowledge management: a selection of methods and techniques. Expert Systems with Applications, v. 13, n. 1, p. 15-27, 1997.

WONG, K. Y.; ASPINWALL, E. Knowledge management implementation frameworks: a review. Knowledge and Process Management, v. 11, n. 2, p. 93$104,2004$.

YIN, R. K. Estudo de caso: planejamento e métodos. Porto Alegre: Bookman, 2005. 